\title{
Optimal Cross-Coupled Synchronizing Control of Dual-Drive Gantry System for a SMD Assembly Machine*
}

\author{
Baeksuk CHU*, Sungsoo KIM**, Daehie $\mathrm{HONG}^{* *}$, Heung-Keun PARK ${ }^{* *}$ and Jinmoo PARK ${ }^{* *}$ \\ The present paper deals with the development of synchronizing controller for dual-drive \\ servo system that is often used for SMD (Surface Mount Device) assembly machine. Instead \\ of coordinating the commands to the individual feed drives and implementing closed position \\ loop control for each axis, this work is achieved by the evaluation of an optimal cross-coupled \\ compensator aimed specifically at improving synchronous accuracy in dual feed drives. The \\ optimal control formulation explicitly includes the synchronous error in the performance in- \\ dex to be minimized. In this paper, surface chip mounter is used for experiment. It demands \\ to synchronize the positions of its two primary driving axes. The system is modeled as the \\ first order approximation and cross-coupled optimal synchronizing controller is designed. \\ The synchronizing control is simulated and experimented with actual system for various ve- \\ locity profiles. The results show that the proposed controller reduces the synchronous error \\ considerably, compared to the conventional uncoupled control for the dual-drive system.
}

Key Words: Synchronous Error, Cross-Coupled Structure, Optimal Control, Performance Index, Multi-Axial Motion Control, Dual-Drive System

\section{Introduction}

In multi-axial motion control systems, for example, manufacturing machine, industrial robot, IC wire bonder, and surface chip mounter, etc., each axis must be controlled accurately and harmoniously to perform the objectives such as precision machining and conveyance. For such purposes, the conventional method is independent axis control, in which the motion of each axis is independently designed and tracked without regard to other axes. In this independent control, if the hardware has an ideal performance by using fast enough controller and well tuned gains, it is possible to achieve relatively good control performance.

A number of researches have been studied about the motion control system. As the independent axis control can achieve the required control objectives by enhancing the tracking ability of each axis, it is possible to apply all kinds of algorithms which can improve the static and dynamic response characteristics of SISO system. Koren ${ }^{(1)}$ has achieved the reduction of the steady state error and overshoot for the first order approximation model of ve-

\footnotetext{
* Received 4th February, 2004 (No. 04-5021)

** Department of Mechanical Engineering, Korea University, 5-1 Anam-dong, Sungbuk-ku, Seoul 136-701, Korea.

E-mail: dhhong@korea.ac.kr
}

locity loop through appropriate gain tuning process. Doraiswami and Gulliver ${ }^{(2)}$ have used the controller which consists of two dynamic elements, a servo compensator and a stabilizing compensator to result in the improvement of the tracking error reduction and the ability removing disturbance. It also gave the robustness about the error near the high frequency band. Tomizuka et al. ${ }^{(3)}$ showed experimentally the preview action to result in large reduction in contour error. Tomizuka also developed a Zero Phase Error Tracking Controller ${ }^{(4)}$, a kind of feedforward control schemes and a repetitive controller ${ }^{(5)}$, a sort of learning control schemes, in the framework of a discrete time control.

However, in the independent axis control, when the dynamic characteristics such as the gain margin and time constant of each axis are different or unsymmetrical disturbance is imposed to the system, the contour error that is defined as the perpendicular distance between the desired path and the real path can be generated. Furthermore, if the tracking performance of each axis is independently enhanced through the improvement of disturbance rejection and the extension of the control bandwidth, the performance is inevitably limited by the stability criterion to decrease the contour error and the saturation of actuator.

In order to alleviate the defects of the independent axis control, the contouring control has been presented. 
In the contouring control, the contour error is directly included in the design of controller and the controller behaves in such a way that it reduces the contour error itself while moving along the desired trajectory. Koren ${ }^{(6)}$ has presented the contouring control for the first time. He used cross-coupled structure to reduce the contour error. Koren and $\mathrm{Lo}^{(7)}$ and Lo and Koren ${ }^{(8)}$ numerically and experimentally showed that including the contour error directly in the design of the controller could result in the improvement of the contour tracking performance compared to the independent axis control when a disturbance occurred to the system or the dynamic characteristics of each axis were not same. Kulkarni and Srinivasan ${ }^{(9)}$ have investigated the cross-coupled compensator scheme in detail. They ${ }^{(10)}$ also introduced the contouring controller using an optimal control algorithm. They presented various performance indices and used an augmented state equation to design the controller along with the appropriately chosen performance index.

In the present paper, we try to minimize the synchronous error for dual drive system, using optimal control with cross-coupled structure. The dual drive system in a gantry structure is an effective way to increase control bandwidth, in which two primary axes aligned in parallel are synchronously driven by identical servo motors. The general contour error is simply transformed into synchronous error in the dual drive system, such that the control problem becomes the efforts to synchronize the motions of both primary axes. We use proportional control algorithm that is a conventional technique in independent axis control and the synchronizing controller is added to provide additional corrective action for specifically improving the synchronizing performance. The controller employs a performance index that explicitly weights on the quadratics of synchronous error and control effort and exhibits cross-coupled structure.

Among the many applications of dual-drive systems, SMD (Surface Mount Device) assembly machine that is often called as surface chip mounter is chosen as a target of control implementation. The synchronizing control is simulated and experimented with actual system for various speed profiles. The performance of the synchronizing control with cross-coupled structure is estimated by comparing to the independent axis control and the crosscoupled control that has been used as a conventional contour control algorithm.

\section{Nomenclature}

Physical scalar quantities are given in italic type, vectors and matrices in bold type.

$\mathbf{A}_{p}$ : coefficient matrix defining state equation

$\mathbf{B}_{p}$ : input coefficient matrix defining state equation

$\mathbf{C}_{p}$ : coefficient matrix

$\mathbf{C}_{r}$ : coefficient matrix $e_{i}$ : position tracking error

$f_{n}$ : frequency of the beam with two torsional springs at the ends

$f_{o}$ : frequency of the beam with clamped ends

$G_{i}(s)$ : velocity loop transfer function

$i$ : number of axis

$J$ : performance index

$K_{p i}, \mathbf{K}_{c}, \mathbf{K}_{p}$ : proportional gain

$K_{p 1}, K_{p 2}$ : gain depending on weighting factor

$K_{v i}$ : velocity loop gain

$n$ : mode number

$p_{i}:$ actual position

$r_{i}$ : weighting factor

$R_{i}, \mathbf{R}$ : reference position

$T$ : sampling time

$u_{i}, \mathbf{u}$ : cross-coupled control input

$V_{c i}, \mathbf{V}_{c}$ : velocity loop control input

$v_{i}:$ actual velocity

$\mathbf{x}_{p}$ : state vector

$\beta$ : stiffness ratio of the joint and the beam

$\varepsilon_{i}, \boldsymbol{\varepsilon}:$ synchronous error

$\tau_{i}:$ time constants

[]$^{T}$ : denotes transpose of a matrix

\section{Dual Drive Mechanism}

A simplified sketch of a typical gantry system is provided in Fig. 1. Two stationary rails aligned in parallel guide the motion of moving beam ( $X$-axis) and this moving beam serves as the guideway for the moving head. Normally, one end of the $X$-axis is driven by a lead (or ball) screw installed on the $Y$-axis and the other end of the $X$-axis passively moves along the opposite $Y$-axis. The driving force is often transferred to the opposite $Y$-axis through appropriate mechanisms but there always exists a deadband between the positions of the primary and the secondary $Y$-axes due to the backlash and/or compliance of these mechanical elements. This type of gantry mechanism is referred to as a 'single drive' mechanism in this paper.

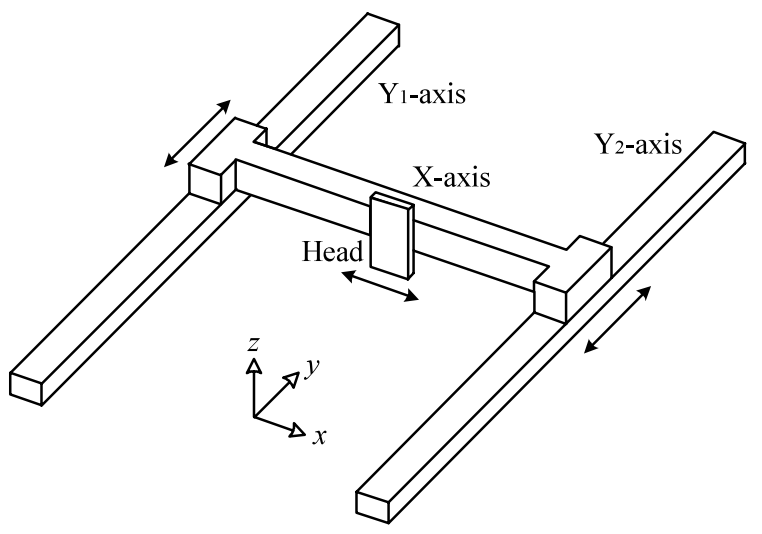

Fig. 1 An $X-Y$ dual-drive gantry mechanism 
(a) Single-drive servo system

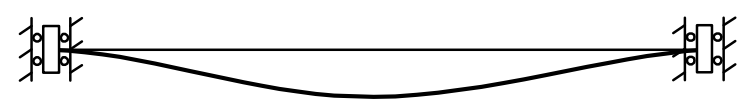

(b) Dual-drive servo system; ideal

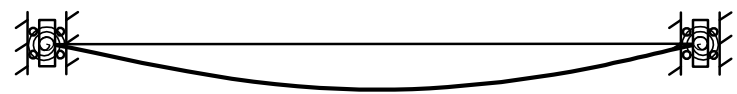

(c) Dual-drive servo system; actual

Fig. 2 Fundamental mode shapes of three different servo configurations

Figure 2 (a) shows the fundamental mode shape of the single drive gantry, which is equivalent to a cantilever beam. The fundamental frequency for a cantilever beam is very low and the system bandwidth is dictated by this mode. One of natural way to increase the bandwidth is shown in Fig. 2 (b), where both ends are fixed to the $Y$ axes that are synchronously driven by identical actuators. In this way, the stiffness of the gantry system greatly increased, so that the control performance can be improved.

However, in case of an abnormal operation or in case that there exist twisting moments due to servo mismatch between the $Y$-axes, hazardous failure can occur at major components. In addition, the friction force exerted on the linear motion guides due to the twisting moments can cause unwanted noise and severe wear. In order to prevent this, compliance mechanisms are often introduced, which can passively absorb the adequate amount of twisting moment. That is, both ends of the X-beam are pin-jointed with torsional springs as shown in Fig. 2 (c). However, adopting the compliance mechanism causes the drawback to decrease the system bandwidth.

The system bandwidth of the dual-drive servo with rigid joints is 6 times bigger than that of single-drive servo system. The modal frequency of the beam with two torsional springs at the ends is given by the following empirical equation ${ }^{(11)}$,

$$
f_{n}=f_{o}\left(\frac{2 n}{2 n+1}\right)^{2}\left[n+\frac{1}{2}\left(\frac{\beta}{5 n+\beta}\right)\right]^{2}
$$

where $f_{o}$ is the frequency of the beam with clamped ends, $n$ is the mode number, and $\beta$ is the stiffness ratio of the joint and the beam. The frequency ratio rapidly increases at low stiffness ratio and approaches to 1 as the stiffness ratio becomes higher. That is, the advantage obtained by increasing the joint stiffness is not so big once it reaches a certain range. For example, the bandwidth will be down over $55 \%$ for the system with springs having negligible stiffness $(n=1, \beta=0)$. However, the shortening of the bandwidth can be reduced up to $20 \%$ with slightly higher stiffness $(n=1, \beta=2)$.

If the synchronous error is minimized, the flexible

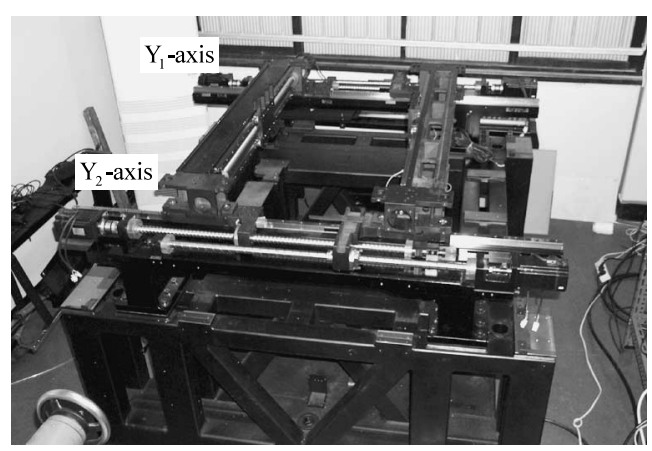

Fig. 3 Picture of dual-drive mechanism for a surface chip mounter

coupling is unnecessary. In this case, high system bandwidth can be chosen and the mechanism design becomes simpler. So, achieving acceptable performance in the synchronizing control gives the advantages on the accurate positioning of the end-effector itself as well as on the system design aspects.

\section{Transfer Function Model}

The target for the control system design and performance evaluation is the surface chip mounter that is widely used to assemble surface mount semiconductor chips on a printed circuit board. This equipment requires very strict synchronization of position controls for dualaxes to avoid servo mismatch. The feed drive system of each axis includes the single-phase AC servomotor, $2.5 \mathrm{~cm} /$ pitch ball screw, and linear guide. The picture in Fig. 3 shows the dual-drive gantry mechanism designed and manufactured for a SMD (Surface Mount Device) chip mounter. It has two sets of dual-gantries installed both of front and back sides of the machine. This is for increasing total productivity by 2 times. They can be considered as identical in control point of view. The feed drive servo for each axis uses SANYO DENKI velocity servo packs. Position sensing is provided by 2000 pulse/rev optical encoders and its pulse signal is multiplied by 4 times after quadrature decoding and sent to up-down counter.

The closed velocity loop was excited by 1volt step input which corresponds to $0.125 \mathrm{~m} / \mathrm{s}$ as a test input and its output was recorded with a data acquisition system. From the experimental data, the approximated model parameters were obtained assuming the first order approximation as follow,

$$
G_{i}(s)=\frac{v_{i}}{V_{c i}}=\frac{K_{v i}}{\tau_{i} s+1} \quad(i=1,2)
$$

$V_{c i}$ and $v_{i}$ are velocity loop input and velocity loop output, respectively. The time constants and velocity loop gains for the primary and secondary axes $\left(Y_{1}\right.$ and $\left.Y_{2}\right)$ were estimated as: $\tau_{1}=0.01153$ [sec], $K_{v 1}=0.9930$ [volt $/$ volt], $\tau_{2}=0.01126$ [sec], and $K_{v 2}=1.0029$ [volt $/$ volt]. The step responses of the first order approximation model are compared to the experimental results in Figs. 4 and 5, respec- 


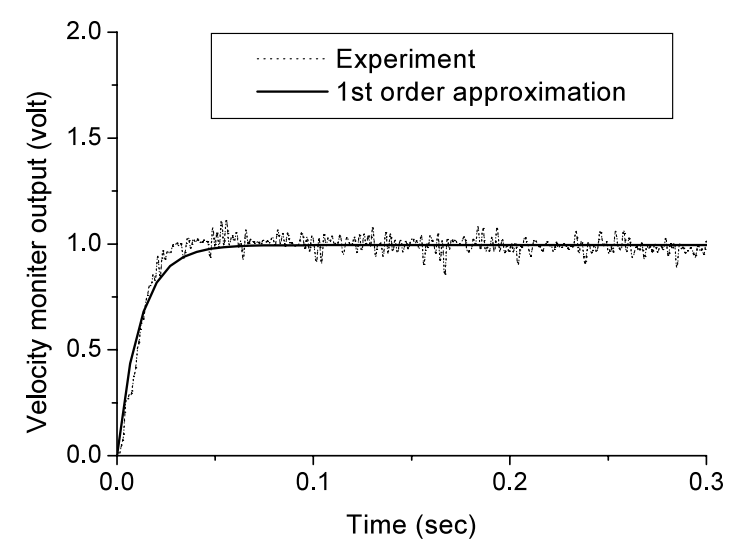

Fig. 4 Step responses of velocity loop of $Y_{1}$-axis: experimental result and 1st order approximation

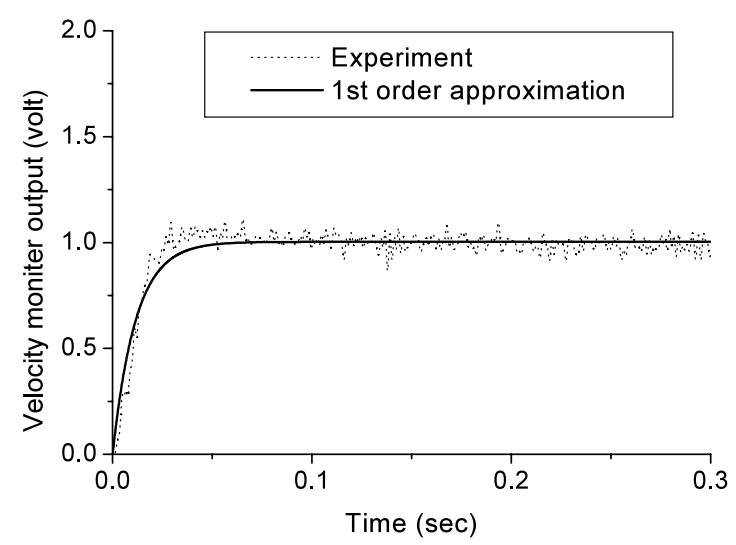

Fig. 5 Step responses of velocity loop of $Y_{2}$-axis: experimental result and 1 st order approximation

tively and it is concluded that the model well represents the target system. Therefore, this first order approximation model will be used for control algorithm development afterward.

\section{Design of Optimal Synchronizing Controller}

The design of optimal control algorithm to improve the synchronizing performance of the dual-servo is described in this section. The optimal control problem formulated here includes the performance index that is a quadratic function of the control error and the crosscoupled control inputs $u_{1}$ and $u_{2}$.

Figure 6 shows the optimal synchronizing control with cross-coupled structure for dual-drive system. In this figure, the position loop for each individual axis constitutes proportional control, whose gains are $K_{p i}(i=1,2)$, as in a conventional structure. The optimal synchronizing control provides an additional control input, $u_{i}(i=1,2)$, to the closed velocity loop of each axis in order to improve the synchronizing performance. Therefore, the control input to the velocity loop, $V_{c i}(i=1,2)$, has the following form:

$$
V_{c i}(k)=K_{p i} e_{i}(k)+u_{i}(k)
$$

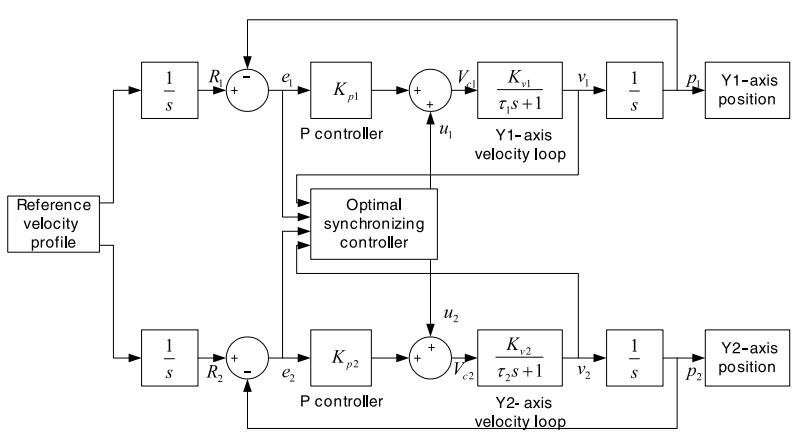

Fig. 6 Block diagram of synchronizing controller including optimal cross-coupled control action

$$
=K_{p i}\left(R_{i}(k)-p_{i}(k)\right)+u_{i}(k),(i=1,2)
$$

where $e_{i}(k),(i=1,2)$ is the position tracking error of each axis that is the difference between the reference position $R_{i}(k),(i=1,2)$ and the actual position feedback $p_{i}(k),(i=$ $1,2)$. An appropriate interpolator generates the reference position at each sampling time based on desired velocity profile.

The difference in the actual position errors of both axes is the synchronous error. In this paper, the synchronous error is defined, such that this difference is equally distributed to each axis, that is,

$$
\boldsymbol{\varepsilon}(k)=\left[\begin{array}{l}
\varepsilon_{1} \\
\varepsilon_{2}
\end{array}\right]=\left[\begin{array}{l}
\frac{1}{2} e_{1}-\frac{1}{2} e_{2} \\
\frac{1}{2} e_{2}-\frac{1}{2} e_{1}
\end{array}\right]
$$

The whole control system can be represented in the state space form with the state vector that is composed of the actual positions $p_{i}(k)$ and its first derivatives $v_{i}(k)$ as follow:

$$
\mathbf{x}_{p}(k+1)=\mathbf{A}_{p} \mathbf{x}_{p}(k)+\mathbf{B}_{p} \mathbf{V}_{c}(k)
$$

where the state vector is

$$
\mathbf{x}_{p}(k)=\left[\begin{array}{llll}
p_{1}(k) & v_{1}(k) & p_{2}(k) & v_{2}(k)
\end{array}\right]^{T}
$$

and the coefficient matrices and the input vector are

$$
\begin{gathered}
\mathbf{A}_{p}=\left[\begin{array}{cccc}
1 & T & 0 & 0 \\
0 & 1-\frac{T}{\tau_{1}} & 0 & 0 \\
0 & 0 & 1 & T \\
0 & 0 & 0 & 1-\frac{T}{\tau_{2}}
\end{array}\right] \\
\mathbf{B}_{p}=\left[\begin{array}{cc}
0 & 0 \\
K_{v 1} \frac{T}{\tau_{1}} & 0 \\
0 & 0 \\
0 & K_{v 2} \frac{T}{\tau_{2}}
\end{array}\right] \\
\mathbf{V}_{c}(k)=\left[\begin{array}{cc}
V_{c 1}(k) & V_{c 2}(k)
\end{array}\right]^{T}
\end{gathered}
$$

The constants $T$ and $\tau_{i}$ are the sampling time and the time constants of the velocity loops, respectively, which 
are used to convert the transfer function (2) into the state space representation.

The synchronous error can be also written as

$$
\boldsymbol{\varepsilon}(k)=\mathbf{C}_{p} \mathbf{x}_{p}(k)+\mathbf{C}_{r} \mathbf{R}(k)
$$

where the coefficient matrices are

$$
\begin{aligned}
& \mathbf{C}_{p}=\left[\begin{array}{cccc}
-\frac{1}{2} & 0 & \frac{1}{2} & 0 \\
\frac{1}{2} & 0 & -\frac{1}{2} & 0
\end{array}\right] \\
& \mathbf{C}_{r}=\left[\begin{array}{cc}
\frac{1}{2} & -\frac{1}{2} \\
-\frac{1}{2} & \frac{1}{2}
\end{array}\right]
\end{aligned}
$$

and the vector for reference trajectory is

$$
\mathbf{R}(k)=\left[\begin{array}{ll}
R_{1}(k) & R_{2}(k)
\end{array}\right]^{T}
$$

Rearranging the control input Eq. (3) leads to the following vector equation:

$$
\mathbf{V}_{c}(k)=-\mathbf{K}_{p} \mathbf{x}_{p}(k)+\mathbf{K}_{c} \mathbf{R}(k)+\mathbf{u}(k)
$$

where the related vector and matrices are

$$
\begin{aligned}
& \mathbf{K}_{p}=\left[\begin{array}{cccc}
K_{p 1} & 0 & 0 & 0 \\
0 & 0 & K_{p 2} & 0
\end{array}\right] \\
& \mathbf{K}_{c}=\left[\begin{array}{cc}
K_{p 1} & 0 \\
0 & K_{p 2}
\end{array}\right] \\
& \mathbf{u}(k)=\left[\begin{array}{ll}
u_{1}(k) & u_{2}(k)
\end{array}\right]^{T}
\end{aligned}
$$

Substituting Eq. (14) into Eq. (5) leads to the following modified state equations.

$$
\mathbf{x}_{p}(k+1)=\left(\mathbf{A}_{p}-\mathbf{B}_{p} \mathbf{K}_{p}\right) \mathbf{x}_{p}(k)+\mathbf{B}_{p} \mathbf{K}_{c} \mathbf{R}(k)+\mathbf{B}_{p} \mathbf{u}(k)
$$

An optimal control approach is formulated for the dual drive system using the state established by the Eqs. (10) and (18), and a synchronizing control input vector $\mathbf{u}(k)$ is obtained in this framework. The following performance index to be minimized is taken,

$$
J=\sum_{k=0}^{\infty}\left[r_{1}\left(\varepsilon_{1}^{2}(k)+\varepsilon_{2}^{2}(k)\right)+r_{2}\left(u_{1}^{2}(k)+u_{2}^{2}(k)\right)\right]
$$

where the first term is an explicit measure of the synchronous error and the second term is a measure of control efforts. The constants $r_{1}, r_{2}$ are weighting factors. The reason to choose this performance index is that the most important control objective for the system is reducing the synchronous error. Further, the control efforts to reach the control objective rather be minimized. In this way, the actuator saturation and the system hunting due to excessive control input signals can be minimized. The control inputs optimally selected with this performance index also reduce the total energy supplied to the controller.

Applying algebraic Riccati equation ${ }^{(12)}$ to these formulations gives the optimal control inputs of the following form,

$$
\begin{aligned}
& u_{1}(k)=K_{p 1} \varepsilon_{1}(k)+\frac{1}{2}\left(K_{D 1} v_{1}(k)-K_{D 2} v_{2}(k)\right) \\
& u_{2}(k)=K_{p 2} \varepsilon_{2}(k)-\frac{1}{2}\left(K_{D 1} v_{1}(k)+K_{D 2} v_{2}(k)\right)
\end{aligned}
$$

where the gains, $K_{p 1}, K_{p 2}, K_{D 1}$, and $K_{D 2}$, depend on the weight factors, $r_{1}, r_{2}$ of the performance index, $J$. The first term includes the synchronous error, $\varepsilon_{i}(i=1,2)$. If the second and third terms are excluded from the formulation, it is identical to the conventional cross-coupled synchronizing control structure. Therefore, the total control inputs consist of the conventional cross-coupled synchronizing control term and the optimal control terms due to the velocity compensation. The optimal synchronizing control structure intends to synchronize the positions of each axis as well as the velocities.

\section{Performance Evaluation and Discussion}

In this section, we discuss the application of the derived control law to the dual-drive position synchronizing control system. The control algorithms are simulated and experimented with actual system for a straight line with trapezoidal speed profile as shown in Fig. 7. In the simulations and experiments, the tracking velocity and acceleration are $1 \mathrm{~m} / \mathrm{sec}$ and $2 \mathrm{G}$, respectively, and the sampling rate is $0.1 \mathrm{msec}$.

In the simulations, the first order approximation models of Eq. (2) which are characterized by the time constants and the velocity loop gains are used for the plant model and the experiments are conducted under the same conditions as the simulations. In this paper, the comparisons are made among three control algorithms; independent axis control, cross-coupled control, and optimal cross-coupled control. In the independent axis control, each axis is controlled independently with proportional gain as in the most of commercial motion control systems. In the crosscoupled control, each axis control is additionally compensated with a proportional control action applied to the synchronous error. That is, the first term in Eqs.(20) and (21) are added to the independent axis control inputs. The optimal cross-coupled control further includes the velocity compensation terms which are the results of optimal control formulation as shown in the second terms of Eqs. (20)

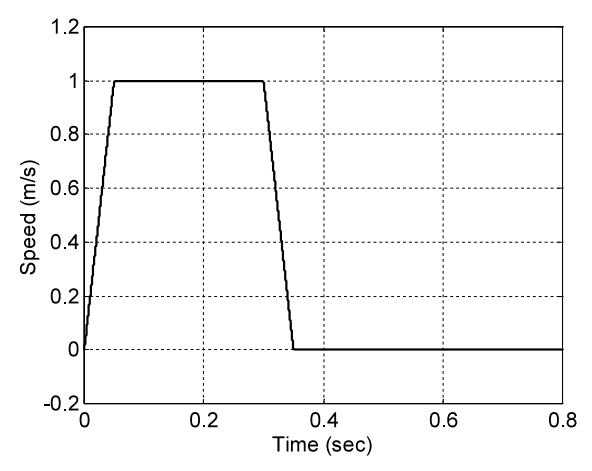

Fig. 7 Trapezoidal reference speed profile 
Table 1 Control gains used in all three algorithms

\begin{tabular}{|c|c|c|c|}
\hline & $\begin{array}{c}\text { independent axis } \\
\text { control }\end{array}$ & $\begin{array}{c}\text { cross-coupled } \\
\text { control }\end{array}$ & $\begin{array}{c}\text { optimal cross-coupled } \\
\text { control }\end{array}$ \\
\hline$K_{p 1}$ & 50 & 50 & 50 \\
\hline$K_{p 2}$ & 50 & 50 & 50 \\
\hline$K_{p 1}$ & 0 & 80 & 80 \\
\hline$K_{P 2}$ & 0 & 80 & 80 \\
\hline$K_{D 1}$ & 0 & 0 & 200 \\
\hline$K_{D 2}$ & 0 & 0 & 200 \\
\hline
\end{tabular}

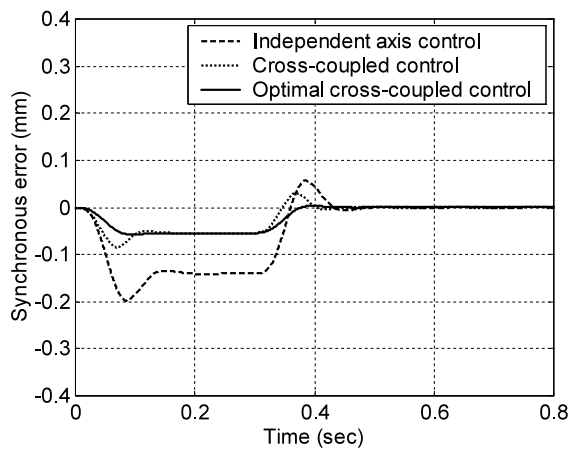

Fig. 8 Simulation results of synchronous error with three different control algorithms

and (21). The control gains used in the simulations for all three algorithms are used for the experiments, which are listed in the Table 1.

The measured parameters in the velocity loops of the real plant already exhibit fair amount of mismatches in the time constants and the loop gains. The computer simulation code was made with these parameters and the responses were obtained as shown in Fig. 8. The time constant discordance causes the synchronous error in transient period, that is, the periods of acceleration and deceleration. The velocity loop gain discordance affects on the steady state error that is distinguished as in the range of constant velocity.

The synchronous error is about 50\% lower both in the cross-coupled control and in the optimal cross-coupled control than in the independent axis control over whole time period. In the optimal cross-coupled control, the additional reduction of synchronous error is observed in the range of acceleration and deceleration. This is because the velocity discordance by the time constant difference is dominant in the transient range and the optimal control input terms in Eqs. (20) and (21) intend to synchronize the velocities of both axes with velocity feedbacks. The experiment also shows the similar result to the simulation as shown in Fig. 9.

The present chip mounter system consists of an $X$ axis, weighing $40 \mathrm{~kg}$, mounted on the dual $Y$-axes and a head, weighing $6 \mathrm{~kg}$, traveling on the $X$-axis. Therefore,

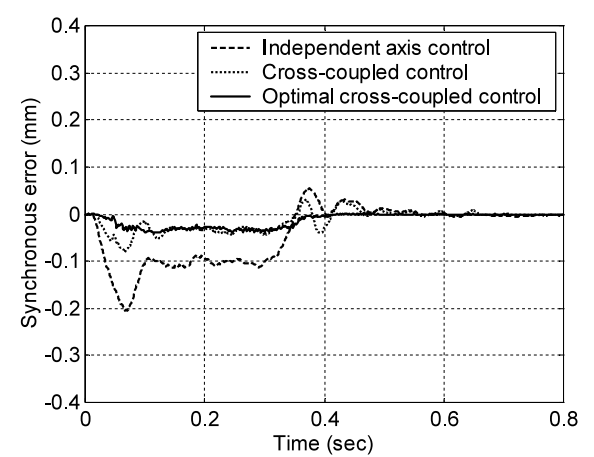

Fig. 9 Experimental results of synchronous error with three different control algorithms

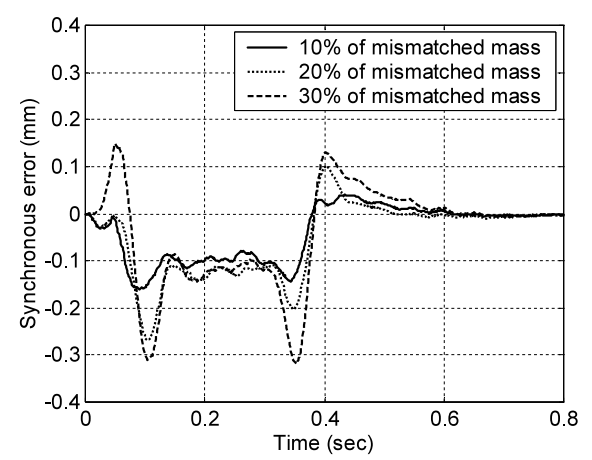

Fig. 10 Synchronous error experimental data for independent axis control in case of $10 \%, 20 \%$ and $30 \%$ mismatched masses

as the position of the head varies along the $X$-axis, the inertia loads due to the weight of the traveling head are differently exerted on each $Y$-drive. This mismatch of inertia loads acts as a major disturbance to the synchronizing controller. In order to investigate the influence of this disturbance to each control algorithms, we intentionally produced the mismatch by adding a weight on one of the $Y$-axes. That is, the weight of $20 \mathrm{~kg}$ was imposed on one $Y$-axis based on the fact that the total weight of $X$-axis was $40 \mathrm{~kg}$. Then, as the additional weights of $2,4,6 \mathrm{~kg}$ were added on one axis, 10, 20, 30\% of mismatches in the inertia forces between two axes were attained.

Three control algorithms were applied to these three different mismatch cases. The experimental results are shown in Figs. 10, 11, and 12. From these figures, it is observed that the synchronous error gradually increases as the weight mismatch increases for all control algorithms. However, the amount of error increase is very small for the case of optimal control algorithm. Also, the disturbance rejection during transient period (acceleration and deceleration periods in the figures) of the optimal control is superior compared to other control algorithms. Consequently, the optimal cross-coupled control exhibits greatly enhanced synchronizing performance both in transient and steady states. 


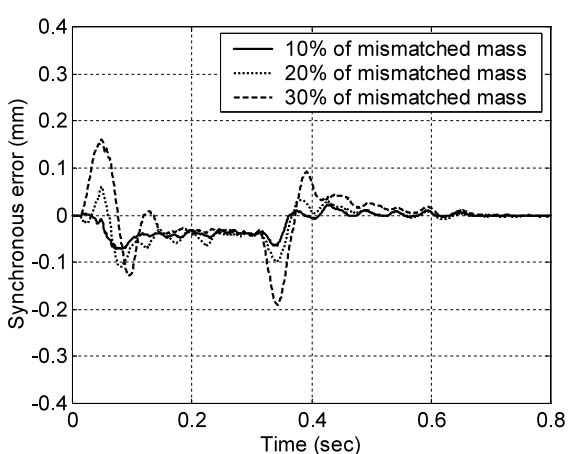

Fig. 11 Synchronous error experimental data for cross-coupled control in case of $10 \%, 20 \%$ and $30 \%$ mismatched masses

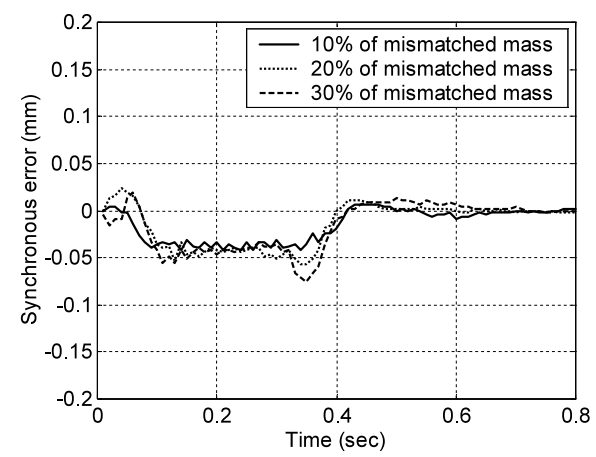

Fig. 12 Synchronous error experimental data for optimal crosscoupled control in case of $10 \%, 20 \%$ and $30 \%$ mismatched masses

\section{Conclusion}

The dual-drive mechanism has been used to increase the system bandwidth of precision gantry systems. This paper describes the development of optimal control algorithm for synchronizing the positions of the dual axes. The cross-coupled control with the synchronizing errors shows good performance in steady state. The additional velocity compensation produced by the optimal control formulation further decreases the synchronizing errors both in transient and steady states. The performance of the developed control algorithm is proved with the application to surface chip mounter through computer simulations and experiments.

\section{Acknowledgment}

This study was supported by the Basic Research
Grants (project number: R01-2002-000-00419-0) supported by Korea Science and Engineering Foundation.

\section{References}

( 1 ) Koren, Y., Computer Control of Manufacturing Systems, (1983), McGraw Hill Book Company.

(2 ) Doraiswami, R. and Gulliver, A., A Control Strategy for Computer of Machine Exhibiting Precision and Rapidity, ASME J. of Dynamic Systems, Measurement, and Control, Vol.106 (1984), pp.56-62.

( 3 ) Tomizuka, M., Dornfeld, D., Bian, X.Q. and Cai, H.G., Experimental Evaluation of the Preview Servo-Scheme for a Two-Axis Positioning System, ASME J. of Dynamic Systems, Measurement, and Control, Vol.106 (1984), pp.1-5.

( 4 ) Tomizuka, M., Zero Phase Error Tracking Algorithm for Digital Control, ASME J. of Dynamic Systems, Measurement, and Control, Vol.109 (1987), pp.65-68.

( 5 ) Tomizuka, M. and Chew, K.K., Digital Control of Repetitive Error in Disk Drive System, Proc. of American Control Conference, (1989), pp.540-543.

( 6 ) Koren, Y., Cross-Coupled Biaxial Computer Control for Manufacturing Systems, ASME J. of Dynamic Systems, Measurement, and Control, Vol.102 (1980), pp.265-272.

( 7 ) Koren, Y. and Lo, C.C., Variable-Gain Cross-Coupling Controller for Contouring, Annals of the CIRP, Vol.40 (1991), pp.371-374.

( 8 ) Koren, Y. and Lo, C.C., Evaluation of ServoControllers for Machine Tools, Proc. of American Control Conference, (1992), pp.370-374.

(9) Kulkarni, P.K. and Srinivasan, K., Cross-Coupled Compensators for Contouring Control of Multi-Axial Machine Tools, Proc. of NAMRC Univ. of Calif. Berkeley, (1985), pp.558-566.

(10) Kulkarni, P.K. and Srinivasan, K., Optimal Contouring Control of Multi-Axial Feed Drive Servomechanisms, ASME J. of Eng. for Industry, Vol.111 (1989), pp.140148.

(11) Park, H.-K., Kim, S.-S., Park, J.-M., Cho, T.Y. and Hong, D., Dynamics of Dual Drive Servo Mechanism, 2001 IEEE Int. Sym. on Industrial Electronics, (2001), pp.1996-2000.

(12) Kwakernaack, Y. and Sivan, R., Linear Optimal Control Systems, (1972), Wiley Inter-Science. 